\title{
Role of alcohol in cancers of the upper alimentary tract: use of models in risk assessment
}

\author{
PERIN N NOTANI \\ From the Epidemiology Unit, Cancer Research Institute, Tata Memorial Centre, Parel, Bombay 400012 , India
}

SUMMARY A case-control study of cancers of the upper alimentary tract was conducted in a hospital to assess the role of several risk factors, including alcohol consumption, which is reported here. Male patients from one community with cancers of the oral cavity $(n=278)$, pharynx $(n=225)$, and oesophagus $(n=236)$ formed the case group. Patients diagnosed as not having cancer $(n=215)$ formed one control group, and a comparable sample of individuals from the general population $(n=177)$ formed another control group. The risk of regular alcohol consumption along with the two well established risk factors of tobacco smoking and chewing were assessed from the linear logistic model fitted. The process of model fitting has been elaborated. Adjusted odds ratios of alcohol consumption in those under 60 years of age varied from 1.3 to 3.6-fold for developing oral cavity cancer, from 1.9 to 5.4-fold for pharyngeal cancer, and from 1.5 to $2 \cdot 7$-fold for oesophageal cancer, in different age groups. No association was observed between alcohol consumption and cancer in those over 60 years of age. A synergistic effect was observed for the combined habit of alcohol drinking with tobacco smoking and/or chewing. The fact that age is a risk factor independent of habit is also demonstrated.

In India, tobacco usage as a risk factor has been well established in several analytical studies of cancers of the upper alimentary 12 and respiratory tracts. ${ }^{34}$ However, no information is available from this region on the risk associated with alcohol consumption and cancer, except for one study on oesophageal cancer. ${ }^{5}$

This paper explores the role of alcohol in cancer of the upper alimentary tract, that is, oral cavity, pharynx, and oesophagus. These cancers form over one-third of all cancers in males, as seen from the incidence figures available from several population based registries in the country, ${ }^{6}$ and the continued exploration of factors associated with these sites seems justified.

\section{Materials and methods}

In a case-control study information on alcohol consumption, which formed a part of the larger study on diet, was obtained from patients coming to Tata Memorial Hospital between 1976 and 1984. The methodology is given in detail elsewhere. ${ }^{7}$ Briefly, cancers of the oral cavity (ICD 8th revision, codes 141, 143-145), pharynx (codes 146,148), and oesophagus (code 150), comprising 278, 225, and 236 cases respectively, treated at this cancer hospital formed the case groups. All the cases were men from one community, viz, Hindus from the State of Maharashtra. Two sets of controls were obtained. One control group was 215 male patients from the same community as the cases, who attended the Head and Neck Unit of the hospital with some complaint but were diagnosed as not having cancer. Futhermore, like the cases, the members of the control group were from the lower socioeconomic strata and received free treatment in the hospital. The second control group of 177 men was from the general population. Bombay city is divided into 170 electoral wards, and electoral rolls are available for each of these wards. A $0.5 \%$ random sample of men was picked from the 1978 electoral rolls of those wards where individuals of socioeconomic levels similar to those of the cases resided. Only those men who belonged to the community under study were interviewed.

Trained interviewers administered a questionnaire, which recorded information on subjects' demographic characteristics, habits of tobacco smoking and chewing, alcohol consumption, usual dietary intake before onset of disease, and medical history. The results with respect to dietary pattern and its association with cancers at these sites has already been reported. ${ }^{7}$ Information on exposure to alcohol and 
tobacco chewing and smoking was obtained in terms of type, frequency, and duration of exposure. Information on the quantity of alcohol consumed was not collected. The analysis is based on the frequency of exposure.

To evaluate the effect of alcohol consumption, it was necessary that the effects of the two well established risk factors of tobacco smoking and chewing were accounted for. The usual method for evaluating the association of alcohol would have been to use the Mantel-Haenszel method ${ }^{8}$ which sums over different strata - here of age and habits - to produce a summary estimate of relative risk associated with alcohol consumption. This method has certain limitations and cannot control for the confounding effects as well as the modifying effects of several variables simultaneously to yield risks associated with each individual variable after adjusting for the effects of all other variables. To overcome this limitation multivariate analysis using loglinear models which were translated into logistic regression models, were resorted to. This method is described in detail by Fienberg. ${ }^{9}$

\section{Results}

The analysis was done for all regular smokers, chewers, and alcohol drinkers. By definition, an individual was considered to be a regular smoker or chewer when the habit occurred at least once a day, and a regular alcohol drinker when consumption was at least once a week. Individuals with less frequent habits than those given above were considered as occasional smokers, chewers, or alcohol drinkers and were not pooled with the group with no habit.

Furthermore, regular smokers could be bidi (obtained by wrapping $0 \cdot 2-0 \cdot 3 \mathrm{~g}$ tobacco in tendu leaf (diospyros melanoxylon)), cigarette or chilum (clay pipe) smokers. Less than $10 \%$ of individuals smoked cigarettes in all groups except population controls $(18 \%)$. Chilum was smoked by an extremely small number of subjects - only nine individuals in the whole data set smoked chilum only. Again, regular chewers were either tobacco chewers (who chewed tobacco with or without other ingredients) or nontobacco chewers (who did not add tobacco to the quid which could consist of several components, viz, betel leaf, betel nut, lime, etc). Non-tobacco chewers varied from $2.3 \%$ and $3.7 \%$ in the two control groups to $4.7 \%$ to $9 \%$ in the case groups.

Those who had regular habits in the past but had stopped a year or more ago were excluded from the group with no habit. The proportion of ex-smokers varied from $5.0 \%$ to $9.7 \%$ for the five groups-the three case and two control groups. Similarly, the proportion of ex-chewers varied from $2 \%$ to $9.7 \%$ and ex-alcohol drinkers from $5 \%$ to $9 \%$.

Table 1 gives the cross classification of all regular alcohol drinkers, regular smokers, regular chewers, and those with no habit by disease status (case/ control), subdivided into two age groups.

The distribution of habits is as one would expect. Considering the two age groups together, it is seen that

Table 1 Cross classification of case and control groups by age and regular* habits

\begin{tabular}{|c|c|c|c|c|c|c|c|c|c|c|}
\hline \multirow[b]{3}{*}{ Case/Control groups } & \multirow[b]{3}{*}{$n$} & \multirow[b]{3}{*}{$\begin{array}{l}\text { Age } \\
\text { group }\end{array}$} & \multicolumn{4}{|c|}{ Regular smoker } & \multicolumn{4}{|c|}{ Non smoker } \\
\hline & & & \multicolumn{2}{|c|}{ Regular chewer } & \multicolumn{2}{|c|}{ Non chewer } & \multicolumn{2}{|c|}{ Regular chewer } & \multicolumn{2}{|c|}{ Non chewer } \\
\hline & & & Alcohol & $\begin{array}{l}\text { No } \\
\text { alcohol }\end{array}$ & Alcohol & $\begin{array}{l}\text { No } \\
\text { alcohol }\end{array}$ & Alcohol & $\begin{array}{l}\text { No } \\
\text { alcohol }\end{array}$ & Alcohol & $\begin{array}{l}\text { No } \\
\text { alcohol }\end{array}$ \\
\hline $\begin{array}{l}\text { Oral cavity } \\
(141,143-145) \dagger\end{array}$ & 217 & $\begin{array}{l}<50 \\
\geqslant 50\end{array}$ & ${ }_{13}^{15}(13)$ & ${ }_{17}^{5}(11)$ & $2^{8}(14)$ & ${ }_{33}^{11}(20)$ & $2^{21}(15)$ & $\begin{array}{l}20 \\
30^{(23)}\end{array}$ & $\begin{array}{l}0^{(0)} \\
0^{(0)}\end{array}$ & $8^{2}$ \\
\hline $\begin{array}{l}\text { Pharynx } \\
(146,148)\end{array}$ & 166 & $\begin{array}{l}<50 \\
\geqslant 50\end{array}$ & ${ }_{16}^{10}(16)$ & $13^{3}(10)$ & $27^{12}(23)$ & $21^{(15)}$ & $1_{10}^{8}$ & $8^{8}(19)$ & $0^{1}$ & $6^{4}(6)$ \\
\hline $\begin{array}{l}\text { Oesophagus } \\
\text { (150) }\end{array}$ & 169 & $\begin{array}{l}<50 \\
\geqslant 50\end{array}$ & $\begin{array}{l}9 \\
14^{(14)}\end{array}$ & ${ }^{3}(12)$ & $2^{8}(18)$ & $2^{6}(17)$ & $8^{6}(8)$ & $21^{11}$ & $\begin{array}{l}1 \\
0\end{array}$ & $13^{(11)}$ \\
\hline Non cancer & 162 & $\begin{array}{l}<50 \\
\geqslant 50\end{array}$ & $3^{3}(4)$ & $3^{6}(6)$ & ${ }_{13}^{6}(12)$ & $9^{5}(9)$ & $5_{13}^{(11)}$ & ${ }_{23}^{30}$ & $0_{0}^{2}(1)$ & ${ }_{18}^{23}(25)$ \\
\hline $\begin{array}{l}\text { Population } \\
\text { Controls }\end{array}$ & 150 & $\begin{array}{l}<50 \\
\geqslant 50\end{array}$ & $\begin{array}{l}0 \\
1\end{array}$ & $3^{2}(3)$ & $7_{11}^{(12)}$ & $12^{11}(15)$ & $5^{5}$ & $\begin{array}{l}10 \\
11\end{array}$ & ${ }_{1}^{3}(3)$ & ${ }_{50}^{18}(45)$ \\
\hline
\end{tabular}

- As defined in the text.

t Codes for International Classification of Diseases, 8th Revision, given in parenthesis under each site. Figures in parenthesis in the body of the table are percent with habits in the (n) individuals of each case/control group. 
the proportion with no habit is higher in the control groups $(25 \%$ and $45 \%)$ and ranges between $5 \%$ and $11 \%$ in the case groups. Furthermore, there are very few individuals who drink alcohol and do not smoke or chew. The proportion with all three habits is low in the control groups ( $1 \%$ and $4 \%)$ whereas in the case groups it ranges from $13 \%$ to $16 \%$.

In the models fitted to these data, age (variable 1) was considered at four levels, viz, $<40$ years, 40 to 49 years, 50 to 59 years, and 60 years and over (levels 1 to 4). Smoking habit (variable 2), chewing habit (variable 3 ), and alcohol consumption (variable 4) were each taken at two levels. Level 1 was for those who had the particular habit (as defined) and level 2 was for those who did not have the habit. Finally, the response variable of disease status (variable 5) was also at two levels: level 1 for case and level 2 for control. Initial analysis was undertaken with non-cancer as the comparison group and for all individuals with regular habits.

There are a number of models to choose from. However, pursuing the research objective of determining the role of alcohol in cancers of the upper alimentary tract, the first model fitted is the one that measures the crude effect of alcohol consumption without taking into consideration the effect of other variables, viz, age and smoking and chewing habits. This is model 1 in table 2 . The second column of the table gives the logistic models fitted.

It is to be expected, and is evident from the $\chi^{2}$ values which are all highly significant, that model 1 does not give a good fit for any site. Introduction of the term for age effect [ ${ }^{\mathrm{u}_{1}}$ (i)] in model 2 adjusts the alcohol effect for the confounding effect of age (because the corresponding term in the loglinear model was the one that measured the interaction effect of age and disease status). Comparison of the $\mathbf{G}^{2}$ statistic with and without the term, for example in the oral cavity group, is $G^{2}(1)-G^{2}(2)=92 \cdot 53-74.83=17 \cdot 70$, which has a chisquare distribution with $27-24=3$ degrees of freedom and is highly significant. Thus it is seen that the term contributes significantly to the model, and hence model 2 has to be chosen over model 1 , and so also for the pharyngeal cancer and oesophageal cancer groups. However, the $\chi^{2}$ values for model-fit for all sites are highly significant so that the overall fit is very poor. In model 3, a term for smoking habit [ ${ }^{\left.\mathrm{H}_{2}(j)\right] \text {, and }}$ in model 4 a term for chewing habit [ $\left.{ }^{3} 3(\mathrm{lc})\right]$ are introduced which measure the extent to which alcohol effect is confounded by these habits. The model-fit has improved with successive additional terms and both these terms contribute significantly to the model, as is evident from the differences in $\mathrm{G}^{2}$ values. In model 5 , the age-alcohol interaction term [ ${ }^{u_{14}}$ (il) $]$ is introduced which measures possible modification of alcohol effect by age, as the corresponding term in the loglinear model measured the degree of association between disease status, alcohol consumption, and age. The differences between the $G^{2}$ values of model 5 and 4 for the three sites $(11 \cdot 87,12 \cdot 46$, and 9.82 as chi-square for $3 \mathrm{df}$ ) are all significant and show that the term contributes significantly to the model. Also the model fit is good as the $p$ values for chi-squares of goodness of fit are $0.21,0.78$, and 0.61 for the three sites, and one selects this model over the preceding ones. In the two subsequent models the terms for modification of alcohol effect by smoking habit and chewing habit are successively introduced and are not significant;

Table 2 Results of models fitted for three sites ${ }^{1}$

\begin{tabular}{|c|c|c|c|c|c|c|c|c|}
\hline \multirow[b]{2}{*}{$\begin{array}{l}\text { Model } \\
\text { (I) }\end{array}$} & \multirow{2}{*}{$\begin{array}{l}\text { Logistic model }{ }^{2} \\
\ln \frac{m_{i j k l 1}}{m_{i j k l 2}}=\end{array}$} & \multirow{2}{*}{$\begin{array}{l}d f \\
\text { with } \\
\text { model } \\
(I)\end{array}$} & \multicolumn{2}{|l|}{ Oral cavity } & \multicolumn{2}{|l|}{ Pharynx } & \multicolumn{2}{|l|}{ Oesophagus } \\
\hline & & & $\chi^{2}(p$ value $)$ & $\begin{array}{l}G^{2}(I)- \\
G^{2}(I+1)^{3}\end{array}$ & $\chi^{2}(p$ value $)$ & $\begin{array}{l}G^{2}(I)- \\
G^{2}(I+1)^{3}\end{array}$ & $\chi^{2}(p$ value $)$ & $\begin{array}{l}G^{2}(I)- \\
G^{2}(I+1)^{3}\end{array}$ \\
\hline 1 & $u+u_{4(1)}$ & 27 & $81.93(<0.001)$ & - & $68.12(<0.001)$ & - & $71.77(<0.001)$ & - \\
\hline 2 & $u+u_{1(i)}+u_{4(1)}$ & 24 & $66.69(<0.001)$ & $17 \cdot 70^{* * *}$ & $51.72(<0.001)$ & $19 \cdot 06^{* * *}$ & $49.54(0.002)$ & $26 \cdot 14^{* * *}$ \\
\hline 3 & $u+u_{1(i)}+u_{2(j)}+u_{4(1)}$ & 23 & $46.08(0.003)$ & $21 \cdot 88^{* * *}$ & $31.88(0.10)$ & $20 \cdot 90^{* * *}$ & $28.35(0.20)$ & $22 \cdot 10^{* * *}$ \\
\hline 4 & $u+u_{1(i)}+u_{2(j)}+u_{3(k)}+u_{4(1)}$ & 22 & $33.12(0.06)$ & $15 \cdot 07^{* * *}$ & $25.97(0.25)$ & $5.91^{*}$ & $25.85(0.26)$ & $3.04+$ \\
\hline 5 & 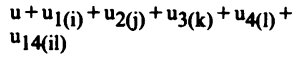 & 19 & $23.56(0.21)$ & $11 \cdot 87^{* * *}$ & $13.99(0.78)$ & $12 \cdot 46^{* * *}$ & $16.73(0.61)$ & $9 \cdot 82^{*}$ \\
\hline 6 & $\begin{array}{l}u+u_{1(i)}+u_{2(j)}+u_{3(k)}+u_{4(1)}+ \\
u_{14(i l)}+u_{24(j l)}\end{array}$ & 18 & $21 \cdot 18(0.27)$ & $2 \cdot 29$ & $13.81(0.74)$ & 0.11 & $16.72(0.54)$ & 0.0 \\
\hline 7 & $\begin{array}{l}u+u_{1(i)}+u_{2(j)}+u_{3(k)}+u_{4(1)}+ \\
u_{14(i l)}+u_{24(j l)}+u_{34(k l)}\end{array}$ & 17 & $20.41(0.25)$ & 0.72 & $13.86(0.68)$ & 0.08 & $16.19(0.51)$ & 0.53 \\
\hline
\end{tabular}

\footnotetext{
1 Comparison group non-cancer cases

2 Variable 1: age (<4v, 40 to 49, 50 to 59, $\geqslant 60$ ); Variable 2: smoking habit (regular, no smoking); Variable 3: chewing habit (regular, no chewing); Variable 4: alcohol intake (regular, no intake); response variable 5 : (1-case, 2-control). Logits for the response variable: $\ln \left(\mathrm{m}_{\mathrm{ijk}} 1 \mathrm{l}^{\mathrm{m}} \mathrm{m}_{\mathrm{ijk}} \mathrm{2}\right)$-Fienberg's $\mathrm{s}^{9}$ notation.

3 The term tested is the one added in model $(I+1)$, with $d f(I)-d f(I+1)$.

$+\mathrm{p}<0 \cdot 10, * \mathrm{p}<0.05, * * \mathrm{p}<0.01, * * \mathrm{p}<0.001$.
} 
Table 3 Estimates of odds ratios associated with regular habits and age obtained under the model fitted, for each of the three sites $^{l}$

\begin{tabular}{|c|c|c|c|c|}
\hline \multicolumn{2}{|c|}{ Adjusted odds ratios ${ }^{2}$ associated with } & Oral Cavity & Pharynx & Oesophagus \\
\hline 1. & Smoking & $4.7 \quad(7.4)$ & $4.5 \quad(5 \cdot 6)$ & $4 \cdot 0 \quad(4 \cdot 7)$ \\
\hline 2. & Chewing & $2.8(11.4)$ & $2 \cdot 1 \quad(6 \cdot 8)$ & $1.6 \quad(4.9)$ \\
\hline 3. & Smoking and chewing & $13 \cdot 1(83 \cdot 5)$ & $9.3(37.8)$ & $6.3(22.8)$ \\
\hline 4. & $\begin{array}{c}\text { Alcohol consumption } \\
\text { in age group }<40 \text { years } \\
40-49 \\
50-59 \\
\geqslant 60\end{array}$ & $\begin{array}{ll} & (1 \cdot 3) \\
3.6 & \\
2.6 & \\
0.9 & \\
0.4 & \end{array}$ & $\begin{array}{ll} & (2 \cdot 2) \\
3 \cdot 4 & \\
3 \cdot 1 & \\
0.4 & \end{array}$ & $\begin{array}{rr}* & \left({ }^{*}\right) \\
2 \cdot 0 & (2 \cdot 7) \\
1.5 & (2 \cdot 6) \\
0.3 & (0 \cdot 6)\end{array}$ \\
\hline 5. & $\begin{array}{cc}\text { Age (years) }^{3} & \\
& <40 \\
& 40-49 \\
& 50-59 \\
& \geqslant 60\end{array}$ & $\begin{array}{ll}1.0 & (1.0) \\
3.3 & (2 \cdot 3) \\
6.1 & (2.9) \\
4.8 & (1.9)\end{array}$ & $\begin{array}{rr}1.0 & (1.0) \\
3.3 & (2.2) \\
5.6 & (2.7) \\
10.5 & (3.0)\end{array}$ & $\begin{array}{ll}1.0 & (1.0) \\
3.8 & (1.8) \\
5.2 & (1.8) \\
9.5 & (3.6)\end{array}$ \\
\hline 6. & Alcohol and chewing & $10.0(15 \cdot 2)$ & $11.6(14.8)$ & $12 \cdot 2(23 \cdot 7)$ \\
\hline 7. & Alcohol and smoking & $17.0 \quad(9.8)$ & $24.6(12 \cdot 3)$ & $30 \cdot 3(22 \cdot 4)$ \\
\hline 8. & Alcohol, smoking, and chewing & $47 \cdot 1\left({ }^{* *}\right)$ & $50 \cdot 2(* *)$ & $47.9(* *)$ \\
\hline
\end{tabular}

- Only 2 oesophageal cancer cases in this age group, with alcohol habit, and therefore odds ratios are not reported.

1 Only 2 oesophageal cancer cases in this age group, with alcohol habit, and therefores ods conts

2 Adjusted odds ratios for combinations of alcohol habit with any other habit (ie, rows 6,7,8) are shown for the youngest age group except the oesophageal cancer group where the odds ratios are given for the next age group, viz, 40 to 49 , for reasons mentioned in $\left(^{*}\right)$

3 Odds ratios associated with age are given for no habit group, except where models with no interaction terms are fitted, ie, figures in parenthesis for oral cavity and pharynx are adjusted (for habits) odds ratios.

** Only 1 individual in the population control group had all 3 habits, therefore the associated odds ratios are not reported.

hence the final model selected is model 5 for all three sites.

When a similar analysis is undertaken with population controls as the comparison group, the best fitting model for the oral cavity and pharynx is model 4 , but for oesophageal cancer model 5 still gives the best fit.

From the expected cell frequencies for the models fitted, one could directly calculate the odds ratios for different covariate combinations of interest. These odds ratios are shown in table 3 . It is seen that almost all the odds ratios calculated under the models fitted are higher when the comparison group is population controls (shown in parenthesis). The first three rows of the table deal with the established risk factors of tobacco smoking and chewing. Odds ratios with respect to smoking habit adjusted for chewing habit, alcohol consumption, and age are shown in the first row for the three sites. These hover between 4 and 5 -fold when the comparison group is non-cancer cases from the hospital and 5 to 7 -fold when the comparison group is from the general population. The next row of the table gives the odds ratios for chewing habit, for the three sites, adjusted for the other variables. The odds ratios, for the combined habit of smoking and chewing are shown next, 13-fold for oral cavity cancers, 9-fold for pharyngeal cancers, and 6-fold for oesophageal cancers, with non-cancer cases as the comparison group, and are higher with the population controls as the comparison group.

Odds ratios associated with alcohol consumption vary with age in models that have age-alcohol interaction term $\left({ }^{u_{14}}\right)$, and these are displayed for individual age groups. Odds ratios for alcohol consumption adjusted for smoking and chewing habits seem to be lower in the older age groups. Excluding the age group 60 and above, the adjusted odds ratios vary with age from 0.9 to $3 \cdot 6$-fold for oral cavity cancers, 1.9 to 5.4 -fold for pharyngeal cancers, and 1.5 to 2.7 -fold for oesophageal cancers in comparison with the two control groups. As there are only two cases of oesophageal cancers in the age group $<40$ years, who consumed alcohol with or without addiction to other habits, the associated odds ratios are not reported.

It should be mentioned that application of the Mantel-Haenszel method $^{8}$ yielded low risks for alcohol consumption (which is not surprising in view of its variation with age). The summary estimates of odds ratios-over strata of age and smoking and chewing habits-together with $95 \%$ confidence intervals were $1.18(0.74,1.88)$ for oral cavity cancers, $1.43(0.86,2.37)$ for pharyngeal cancers, and 1.05 $(0.62,1.76)$ for oesophageal cancers with non-cancer cases as the controls. Similar low risks $(1.17,1.99$, and 1.34 respectively) were also obtained with population controls as the comparison group. 
Age as a risk factor irrespective of the three habits is also demonstrated. The odds ratios associated with age in models with interaction term are shown for the no habit group only in the table. Thus if the risk of an individual under $\mathbf{4 0}$ years of age who does not smoke, chew or drink alcohol is unity, then for a similar individual in the age group 50-59, say, the risk goes up 5 to 6-fold for different sites with non-cancer cases as the comparison group. With no interaction term in the models fitted to oral cavity and pharyngeal cancers and population controls as the comparison group, the adjusted odds ratios go up 2 to 3 -fold with increase in age, independent of the three habits.

The risks for the combination of habits can also be obtained from the odds ratios of individual variables. Hence the odds ratios associated with combinations involving alcohol consumption have been shown for only one age group in table 3 : for oral cavity cancers and pharyngeal cancers the youngest age group $(<40$ years) and for oesophageal cancers the next age group (40-49 years), for reasons already mentioned. In general the highest risk is seen for those who have all three habits of smoking, chewing, and alcohol drinking except for the age group 60 and above, where the combination of smoking and chewing carries the highest risk for each site.

The analysis was repeated, using only regular bidi smokers instead of all smokers and those who chewed tobacco instead of all chewers, with the non-cancer cases as the comparison group. There was no appreciable difference in the odds ratios associated with different habits and hence those have not been reported separately. This may well be because $80 \%$ of all regular smokers were bidi smokers and $90 \%$ of all regular chewers were tobacco chewers, ie, chewed tobacco with or without other ingredients, in the whole data set.

It would have been of interest to assess the doseresponse relation with quantity or with frequency of alcohol consumption but that was not possible because no information was collected on the quantity of alcohol consumed by the subjects, and the numbers per cell reduced sharply when the break-up by frequency of intake was considered.

\section{Discussion}

In this study alcohol drinking is shown to be a risk factor for cancers of the upper alimentary tract, in addition to smoking and chewing habits.

Almost all who drank alcohol consumed what is known as 'country' liquor - a locally distilled brewexcept for 12 individuals in the whole data set who drank whisky, rum or beer. The risk associated with alcohol drinking was higher in the younger age groups, and no association was seen in the age group 60 years and above. In fact the proportion of regular alcohol consumers was much lower in the age group 60 and above, varying from $19 \%$ to $24 \%$ for the three sites, whereas in the cases under 60 years of age the proportion of alcohol consumers was $38 \%$ to $48 \%$ for the three sites. In the under 60 age groups the risk of alcohol consumers developing oral cavity cancers went up to 3.6 -fold, for pharyngeal cancers 1.9 to 5.4-fold, and for oesophageal cancers 1.5 to $2 \cdot 7$-fold after adjusting for smoking and chewing habits. The risks were higher when alcohol was drunk in conjunction with tobacco chewing and/or smoking.

There is only one study from this region ${ }^{5}$ which reports risks associated with alcohol consumption and oesophageal cancers. In this study a 12 -fold risk was shown for men who drank alcohol as well as chewed tobacco, and 18-fold for those who both smoked and drank alcohol. The risks for these combinations in the present study are similar or slightly higher. The risk of alcohol consumers and chewers for developing oesophageal cancers was 12 to 23-fold, except in the $60+$ age group and for alcohol consumers and smokers the risk varied from 10 to 30 -fold in different age groups, and with the two control groups. The earlier study ${ }^{5}$ was unable to demonstrate an association of alcohol intake alone with oesophageal cancers, whereas it has been possible to do so in the present study (1.5 to 2.7 -fold, varying with age and two control groups).

The relation between alcohol and cancer has been known for some decades. Several case-control studies have demonstrated an increase in cancers of the upper alimentary tract in those who consume large amounts of alcohol in conjunction with cigarette smoking.

A study ${ }^{10}$ in a population (Puerto Rican) economically similar to ours and with high incidence rates of cancers at these sites has reported risks for alcohol consumers - mainly illicitly distilled rumafter adjusting for smoking habit as 1.57 for mouth cancers, 1.62 for pharyngeal cancers, and 2.85 for oesophageal cancers. Another case-control study in Singapore Chinese ${ }^{11}$ has reported risks associated with samsu drinking for oesophageal cancers. Samsu is Chinese wine and is reported to be a strong liquor, the alcohol content being equivalent to that of whisky. The risk for samsu consumers was significant at 1.97, and went up to 2.87 in those who drank daily. However, when this was adjusted in a logistic regression model, for several factors including smoking the risk was reduced to $\mathbf{1} \cdot 20$.

The risk of mouth and pharyngeal cancers in American whites has been reported ${ }^{12}$ to range between 2.6 and 3.7 in those who smoke cigarettes and drink wine, whisky or beer, and the risk of oesophageal cancer ${ }^{13}$ to be $6 \cdot 4$-fold in heavy smokers and whisky drinkers. 
Alcohol drinking has also been shown ${ }^{14}$ to be a risk factor independent of cigarette smoking in cancers of the mouth and pharynx. The risk increased from 1.4 to 2.3-fold as the amount of alcohol intake increased from less than $0.4 \mathrm{oz}$ to more than $1.5 \mathrm{oz}$ a day, and in heavy smokers and alcohol drinkers the risk went up to 15-fold compared to those who neither smoked nor drank. Similarly, for cancers of the oesophagus, a study from France ${ }^{15}$ reported a risk up to 18 -fold in heavy alcohol consumers ( $\geqslant 81 \mathrm{~g} /$ day) and 44-fold in those who were heavy smokers ( $\geqslant 20$ cigs/day) as well as heavy alcohol drinkers.

Thus several studies have demonstrated the association not only of alcohol consumption together with cigarette smoking, but also of alcohol drinking per se, with cancers of the upper alimentary tract. Nevertheless, it is unlikely that pure ethyl alcohol would be directly carcinogenic to man since animal experiments have invariably been negative. Probably it is not alcohol per se but its contaminants that may be carcinogenic.

Furthermore, nutritional deficiencies as mentioned in several studies may also increase susceptibility to the carcinogenic potential of an external agent. The increased risk of cancers at these sites (adjusted for habits) associated with poor nutrition, in particular the lack of fresh vegetables, fish, and to some extent pulses and buttermilk in the diet of cases before disease onset, has already been reported. ${ }^{7}$

It should be mentioned that the case group and non-cancer control group were economically comparable and were picked from patients who were treated free in the hospital. Efforts were made to select general population controls from the same socioeconomic strata as the cases. Nevertheless, it needs to be noted that this was a purely urban group as opposed to a mix of urban-rural residents in the case group. This may give rise to case-control differences with respect to some likestyle factors that were not controlled for in the analysis.

Despite the limitations of a case-control approach, the present study has identified alcohol drinking as one of the risk factors that may be responsible for the elevated risk of upper alimentary tract cancers in men of the population studied. However, its mode of action in the carcinogenesis process is not clear and remains to be resolved. The analytical method employed has been elaborated, illustrating one of the frequently used and relatively newer techniques of model fitting, which is applied to assess relationships between several variables and their effects on disease outcome.
I would like to thank the management of Tata Memorial Hospital for permission to interview the patients and also for granting access to their medical records; Dr T D Koepsell, Veterans Administration Medical Center, Seattle, Washington, USA, for providing the generic Microsoft BASIC program for loglinear analysis, which was modified to run on the Commodore-64. Thanks are also due to Mrs Usha Dighe for her patient and painstaking interviewing of the patients throughout the period of the study.

\section{References}

${ }^{1}$ Sanghvi LD, Rao KCM, Khanolkar VR. Smoking and chewing of tobacco in relation to cancer in upper alimentary tract. $\mathrm{Br}$ Med J 1955; 1: 1111-4.

2 Jussawalla DJ, Deshpande VA. Evaluation of cancer risk in tobacco chewers and smokers. An epidemiological assessment. Cancer 1971; 28: 244-52.

${ }^{3}$ Notani PN, Sanghvi LD. A retrospective study of lung cancer in Bombay. Br J Cancer 1974; 29: 477-82.

4 Jussawalla DJ, Jain DK. Lung cancer in Greater Bombay, correlation with religion and smoking habits. Br J Cancer 1979; 40: 437-48.

5 Jussawalla DJ. Epidemiological assessment of aetiology of oesophageal cancer in Greater Bombay. Monograph, International Seminar on Epidemiology of Esophageal Cancer 1971; 1:20-30.

${ }^{6}$ Annual Report 1984, of National Cancer Registry: A project of Indian Council of Medical Research, Ansari Nagar, New Delhi; prepared by co-ordinating Unit of National Cancer Registry at Bombay.

${ }^{7}$ Notani PN, Jayant K. Role of diet in upper aerodigestive tract cancers. Nutrition and Cancer 1987; 10: (1/2): 103-13.

${ }^{8}$ Mantel N, Haenszel W. Statistical aspects of the analysis of data from retrospective studies of disease. JNCI 1959; 22: 719-48.

${ }^{9}$ Fienberg SE. The analysis of cross-classified categorical data. Cambridge, MA: MIT Press, 1980.

10 Martinez I. Factors associated with cancer of the oesophagus, mouth and pharynx in Peurto Rico. JNCI 1969; 42: 1069-94.

${ }^{11}$ De Jong UW, Breslow N, Hong GE, Sridharan M, Shanmugaratnam K. Aetiological factors in oesophageal cancer in Singapore chinese. Int J Cancer 1974; 13: 291-303.

12 Wynder EL, Bross IJ, Feldman RM. A study of the etiological factors in cancer of the mouth. Cancer 1957; 10: 1300-23.

${ }^{13}$ Wynder EL, Bross IJ. A study of etiological factors in cancer of oesophagus. Cancer 1961; 14: 389-413.

14 Rothman K, Keller AZ. The effect of joint exposure to alcohol and tobacco on risk of cancer of mouth and pharynx. J Chron Dis 1972; 25: 711-6.

15 Tuyns AJ. The cause of cancer: Alcohol; In Cancer epidemiology and prevention, eds D Schottenfeld and J Fraumeni Jr. Philadelphia PA: Saunders: 1982; 293-303.

Accepted for publication February 1988 